\title{
Tratamento endodôntico durante os períodos de gestação e amamentação: uma
}

\section{revisão integrativa da literatura}

\author{
Endodontic treatment during pregnancy and breastfeeding periods: an integrative literature review \\ Tratamiento de endodoncia durante el embarazo y la lactancia: una revisión integradora de la
}

\section{Resumo}

A gestação é um período fisiológico complexo, com alterações hormonais que geram mudanças físicas e emocionais no corpo da mulher. Portanto, a atuação do cirurgião dentista nesta etapa é fundamental, principalmente, colaborando por circunstâncias mais segura para o nascimento da criança. Diante disso, o objetivo dessa revisão integrativa é realizar um levantamento bibliográfico sobre os principais fatores e cuidados relacionados ao tratamento endodôntico nos períodos de gestação e amamentação. A questão norteadora do estudo foi: "Quais os principais fatores e cuidados relacionados ao tratamento endodôntico nos períodos de gestação e amamentação?”. As buscas foram realizadas nas bases de dados PubMed/MEDLINE, Lilacs e SciELO até setembro de 2021. No total 10 estudos publicados entre 2008 e 2020 foram incluídos nessa revisão integrativa. Foi possível concluir que o tipo de anestésico local, tomadas radiográficas e a terapia medicamentosa são fatores críticos para um tratamento sem riscos a mãe e o bebê.

Palavras-chave: Endodontia; Gravidez; Aleitamento materno; Complicações na gravidez.

\begin{abstract}
Pregnancy is a complex physiological period, with hormonal changes that generate physical and emotional changes in the woman's body. Therefore, the role of the dental surgeon at this stage is essential, mainly, contributing to safer circumstances for the child's birth. Therefore, the objective of this integrative review is to carry out a literature review on the main factors and care related to endodontic treatment during pregnancy and breastfeeding. The guiding question of the study was: "What are the main factors and care related to endodontic treatment in the periods of pregnancy and breastfeeding?". Searches were performed in PubMed/MEDLINE, Lilacs and SciELO databases until September 2021. A total of 10 studies published between 2008 and 2020 were included in this integrative review. It was possible to conclude that the type of local anesthetic, radiographic measurements and drug therapy are critical factors for a risk-free treatment for mother and baby.
\end{abstract}

Keywords: Endodontics; Pregnancy; Breast feeding, Pregnancy complications.

\section{Resumen}

El embarazo é um período fisiológico completo, com mudanças hormonais que geram mudanças físicas e emocionais no cuerpo de la mujer. Por tanto, o papel do cirujano dentista nesta etapa é fundamental, principalmente, contribuindo com as circunstâncias, mas seguras para o nacimiento del niño. Por tanto, o objetivo de esta revisão integradora é realizar uma revisão da literatura sobre os principais fatores e cuidados relacionados com o tratamento endodôntico durante o embarazo e a lactância. La pregunta orientadora del estudio fue: “¿Cuáles son los principales fatores y cuidados relacionados con el tratamiento de endodoncia durante os períodos de embarazo y lactancia? ". Las búsquedas se realizar nas bases de dados PubMed / MEDLINE, Lilacs e SciELO hasta setembro de 2021. En esta revisión integradora se incluyeron um total de 10 estudos publicados entre 2020 y 2008 . Se pudo terminar que o tipo de anestésico local, las mediciones radiográficas e a farmacoterapia são fatores obrigatórios para um tratamento sem riesgo para a mãe e o bebê.

Palabras clave: Endodoncia; Embarazo; Lactancia materna; Complicaciones del embarazo. 


\section{Introdução}

A gestação é um período fisiológico complexo, com alterações hormonais que geram mudanças físicas e emocionais no corpo da mulher, assim exige dos profissionais da saúde conhecimentos específicos para uma assistência mais integral (Codata et al, 2011). A atenção odontológica neste período, na maioria das vezes, é negligenciada devido crenças e mitos envolvendo a saúde mãe-filho, gerando uma série de dúvidas no profissional e no paciente, referentes aos procedimentos que podem ser executados (Codata et al, 2011; Miana et al, 2010).

Durante a gestação, a mulher se encontra mais perceptiva a novos conhecimentos relacionados a sua saúde e a saúde do seu bebê, além disso, torna-se multiplicadora de informações para todo seu núcleo familiar, sendo esta uma etapa favorável para promoção de saúde (Cabral et al, 2013). Portanto, a atuação do cirurgião dentista nesta etapa é fundamental, principalmente, colaborando por circunstâncias mais segura para o nascimento da criança (Martins et al, 2013; Praetzel et al, 2010).

A Saúde Pública no Brasil trabalha a promoção de saúde aliada ao preceito odontológico como parte da integração de saúde da família, sendo recomendado pelo Ministério da Saúde (MS) que a gestante seja encaminhada ao cirurgião dentista para receber orientações sobre a possibilidade de atendimento durante a gestação, exames de tecidos moles, identificação de risco à saúde bucal, diagnóstico de lesões de cárie, necessidade de tratamento curativo, diagnóstico de gengivite ou doença periodontal crônica, necessidade de tratamento e orientações sobre hábitos alimentares e higiene bucal (Braz et al, 2010; Ministério da Saúde, 2012).

Evidencias científicas comprovam a correlação entre doenças da cavidade bucal e o período da gestação, que podem desencadear desde o parto prematuro e baixo peso ao nascer até a transmissão de Streptococos mutans via mãe-filho (Catão et al, 2015; Reis \& Almeida, 2013; Codata et al, 2011). A doença periodontal crônica são frequentes em gestantes, devido aos altos índices de estrógeno e progesterona, sendo este um fator de relação direta à partos prematuros. A doença cárie também são frequentes, devido ao aumento de uma alimentação cariogênica, queda da capacidade de tampão da saliva e deficiência na higienização bucal (Botelho et al, 2019).

O primeiro trimestre da gestação se mostra o período menos adequado para o tratamento odontológico, pois ocorrem as principais transformações embriológicas, sendo principalmente evitado as tomadas radiográficas. O segundo trimestre é o mais indicado para intervenções odontológicas, pois é o período que a organogênese está completa e o feto desenvolvido, além de ser o período mais confortável para a mãe quando comparado aos estágios iniciais e finais da gestação (Botelho et al, 2019). No entanto, procedimentos de urgência odontológica, como procedimentos endodônticos, não são contraindicados de serem realizados no primeiro trimestre, principalmente em casos de dor e infecção local (Jain et al, 2015; Kandan et al, 2011).

A saúde bucal da gestante ainda é cercada de muitas dúvidas e crenças passadas ao longo dos tempos por meio de experiências relatadas por familiares e outras gestantes, sendo o medo um dos principais fatores que contribuem para o afastamento da gestante à atenção odontológica (Botelho et al, 2019). Além disso, existe a hesitação em tratar pacientes gestantes por parte dos cirurgiões-dentistas, sendo isso atribuído, principalmente, à formação acadêmica, visto que raras são as universidades que possuem em sua grade curricular a possibilidade de um estudo direcionado ao atendimento de gestantes e mães em períodos de amamentação (Botelho et al, 2019; Braz et al, 2010).

Diante disso, o objetivo dessa revisão integrativa é realizar um levantamento bibliográfico sobre os principais fatores e cuidados relacionados ao tratamento endodôntico nos períodos de gestação e amamentação. 


\section{Metodologia}

Trata-se de uma Revisão Integrativa da Literatura, compreendida como um método caracterizado pelo interesse do pesquisador em sumarizar resultados de um conjunto de pesquisas sobre um mesmo tema, permitindo o vínculo entre as evidências de pesquisas e as práticas de saúde, com possibilidade de contribuição aos cuidados prestados aos usuários dos serviços de saúde (Mendes et al, 2008). O estudo será desenvolvido nas seguintes etapas: identificação do tema e elaboração da questão norteadora do estudo; estabelecimento de critérios de inclusão e exclusão de estudos; realização de buscas na literatura científica; definição das informações a serem coletadas e avaliação dos estudos selecionados para a revisão; interpretação; e síntese do conhecimento.

A questão norteadora do estudo foi: "Quais os principais fatores e cuidados relacionados ao tratamento endodôntico nos períodos de gestação e amamentação?". As buscas foram realizadas nas bases de dados PubMed/MEDLINE, Lilacs e SciELO até setembro de 2021. Foi utilizado descritores presentes no Medical Subject Headings (MeSH) para as buscas no PubMed/MEDLINE e descritores presentes no Descritores em Ciências da Saúde (DeCS) para as buscas nas plataformas Lilacs e SciELO, assim os seguintes descritores foram empregados: "gravidez” (DeCS) "pregnancy” (MeSH) "amamentação" (DeCS) “breast-feeding” (MeSH) “complicações na gestação” (DeCS) “pregnancy complications” (MeSH) “tratamento odontológico" (DeCS) “dental anxiety” (MeSH) “endodontia” (DeCS) “endodontics” (MeSH). Os descritores foram associados entre si com o emprego do operador booleano AND e OR.

Foi construído os seguintes critérios de inclusão: diretrizes de práticas clínicas relatando tratamento endodôntico em grávidas, publicados em português, inglês ou espanhol, com texto completo e disponível na íntegra nas bases de dados selecionadas, publicados até setembro de 2021. Os critérios de exclusão foram: artigos que não utilizavam do tratamento endodôntico e/ou pacientes não grávidas, artigos duplicados nas bases de dados com texto incompleto ou indisponível na íntegra, e artigos que não se enquadravam na temática e no objetivo do estudo.

Para avaliação dos dados foi elaborado um instrumento de pesquisa, estruturado de acordo com a questão norteadora do estudo, dividido em onze itens destinados ao registro das informações dos artigos: numeração, título, identificação dos autores, titulação dos autores, ano de publicação, periódico, objetivo do estudo, metodologia, local do estudo, resultados e conclusões. Esse instrumento foi preenchido após a leitura do conteúdo dos artigos, tendo em vista a questão norteadora do estudo.

Para análise e interpretação dos dados elaborou-se um quadro sinóptico destinado ao registro da síntese das informações obtidas nos artigos. Esses elementos serviram de subsídios para a análise e discussão dos resultados e a resposta ao problema de pesquisa.

Para analisar os dados, ocorreu a avaliação inicial dos artigos selecionados, os mesmos foram comparados entre si, buscando-se dialogar com as semelhanças e pontuar as diferenças. Após, os dados obtidos foram registrados na forma de quadro sinóptico, que possibilitou uma interpretação mais sintetizada e comparativa dos dados encontrados.

\section{Resultados}

A pesquisa nos bancos de dados selecionou 239 artigos, incluindo 171 estudos no PubMed/MEDLINE, 6 estudos no Lilacs e 62 SciELO. Todas as referências duplicadas foram excluídas e realizado a aplicação dos critérios de inclusão/exclusão, o que resultou no quantitativo de 10 artigos. Os resultados encontrados nas bases de dados estão dispostos na Tabela 1. 
Tabela 1 - Distribuição das referências bibliográficas encontradas e selecionadas de acordo com as bases de dados e os descritores utilizados.

\begin{tabular}{ccc}
\hline Dados de Bases & $\begin{array}{c}\text { Estudos } \\
\text { Encontrados }\end{array}$ & $\begin{array}{c}\text { Estudos } \\
\text { Selecionados }\end{array}$ \\
\hline PubMed & 171 & 7 \\
Lilacs & 6 & 0 \\
SciELO & 62 & 3 \\
Total & 239 & 10 \\
\hline
\end{tabular}

Fonte: Autores.

No total 10 estudos publicados entre 2008 e 2020 foram incluídos nessa revisão integrativa. As características dos estudos incluídos estão listadas na Tabela 2.

Tabela 2 - Distribuição dos estudos selecionados de acordo com autores, ano de publicação, número de pacientes e conclusão.

\begin{tabular}{|c|c|c|}
\hline Autor / Ano & Tema Abordado & Conclusão \\
\hline $\begin{array}{l}\text { Zanata et al, } \\
\quad 2008 \text {. }\end{array}$ & Manejo & $\begin{array}{c}\text { O manejo odontológico na gestação ainda apresenta alguns desvios } \\
\text { das recomendações da literatura científica }\end{array}$ \\
\hline $\begin{array}{l}\text { Michalowicz et } \\
\text { al, } 2008 .\end{array}$ & Complicações e Manejo & $\begin{array}{l}\text { Tratamento odontológico entre } 13 \text { e } 21 \text { semanas de gestação não foi } \\
\text { associado a um risco aumentado de ocorrência de eventos adversos } \\
\text { médicos graves }\end{array}$ \\
\hline $\begin{array}{l}\text { Lopes et al, } \\
2008 \text {. }\end{array}$ & $\begin{array}{c}\text { Uso de anestésico local e } \\
\text { Medicação }\end{array}$ & $\begin{array}{c}\text { Penicilinas foi a } 1^{\circ} \text { escolha para as gestantes, assim como o } \\
\text { Paracetamol constitui o analgésico mais indicado. Lidocaína } \\
\text { associada a agentes vasoconstritores e também foram estes que se } \\
\text { destacaram }\end{array}$ \\
\hline $\begin{array}{l}\text { Giglio et al, } \\
2009 .\end{array}$ & Complicações e Manejo & Diretrizes são necessárias para melhor manejo de complicações \\
\hline $\begin{array}{l}\text { Amadei et al, } \\
2011 .\end{array}$ & $\begin{array}{l}\text { Terapêutica } \\
\text { medicamentosa em } \\
\text { gestantes e lactantes }\end{array}$ & $\begin{array}{c}\text { Terapêutica medicamentosa em gestantes e lactantes requer sempre } \\
\text { uma avaliação cuidadosa dos riscos potencialmente envolvidos } \\
\text { nesta prática }\end{array}$ \\
\hline Luc et al, 2012. & Uso de anestésico local & Anestesia dental não é contraindicado \\
\hline $\begin{array}{l}\text { Donaldson \& } \\
\text { Goodchild, } \\
2012 \text {. }\end{array}$ & $\begin{array}{c}\text { Uso de anestésico local e } \\
\text { Medicação }\end{array}$ & $\begin{array}{l}\text { Em alguns casos, a dosagem da medicação deve ser evitada ou } \\
\text { alterada; entretanto, há momentos em que é desnecessário } \\
\text { interromper o uso de medicamentos }\end{array}$ \\
\hline $\begin{array}{l}\text { Ebrahim et al, } \\
2014 .\end{array}$ & $\begin{array}{c}\text { Uso de anestésico local e } \\
\text { Medicação }\end{array}$ & $\begin{array}{c}\text { A amoxicilina e o paracetamol são os medicamentos de escolha } \\
\text { quando necessários, assim como a utilização da lidocaína com } \\
\text { epinefrina nas anestesias locais }\end{array}$ \\
\hline $\begin{array}{l}\text { Dalto et al, } \\
2017 .\end{array}$ & $\begin{array}{c}\text { Uso de anestésico local e } \\
\text { Medicação }\end{array}$ & $\begin{array}{c}\text { O uso de anestésicos embora tenham a capacidade de atravessar a } \\
\text { placenta, apresentam segurança no uso, já alguns fármacos podem } \\
\text { apresentar restrições trazendo possíveis complicações durante o } \\
\text { período gestacional }\end{array}$ \\
\hline $\begin{array}{l}\text { Ather et al, } \\
2020 \text {. }\end{array}$ & $\begin{array}{c}\text { Terapêutica } \\
\text { medicamentosa }\end{array}$ & $\begin{array}{l}\text { O tratamento endodôntico pode e deve ser fornecido de forma } \\
\text { segura e rotineira a mulheres grávidas. }\end{array}$ \\
\hline
\end{tabular}

Fonte: Autores. 


\section{Discussão}

O procedimento endodôntico demanda do uso de anestésico local, sendo anestésicos com vasoconstritores de primeira escolha ao profissional. Entretanto, no caso de pacientes grávidas essa é uma etapa bastante crítica que exige conhecimento específico do profissional, em vista que anestésicos com vasoconstritores de felipressina, presente no anestésico da prilocaína, podem ser um grande risco para a vida do feto, pois sua estrutura é similar ao hormônio da citocina que em grandes concentrações podem induzir a contração uterina, e consequentemente, causar um aborto. Assim, sempre que possível, o cirurgião-dentista deve utilizar anestésicos locais com vasoconstritor de epinefrina (Dalto et al, 2017; Ebrahim et al, 2014; Luc et al, 2012). A lidocaína na concentração $1: 100.000$ a $2 \%$, que tem a epinefrina como vasoconstritor, é o anestésico local de primeira escolha nos atendimentos a mulheres grávidas (Amadei et al, 2011). Além disso, o uso da lidocaína é liberado para mães em períodos de amamentação, pois mesmo sendo excretada no leite, as concentrações são pequenas e não causam prejuízo ao bebê (Ebrahim et al, 2014). O uso de Mepivacaína e Bupvacaína devem ser administradas com cautela, pois a alta concentração e sua rápida ação podem causar uma bradicardia no feto (Dalto et al, 2017; Ebrahim et al, 2014).

$\mathrm{O}$ uso de tomadas radiográficas também é necessário durante o procedimento endodôntico, porém, muitos profissionais apresentam receios em utilizar em pacientes gestantes devido à radiação. No entanto, estudos vêm demonstrando que o risco teratogênico da exposição à radiação dos filmes intraorais é 1000 vezes menor do que o risco natural de uma mulher ter aborto espontâneo ou malformação fetal $(<0.1 \%)$ (Amadei et al, 2011; Kandan et al, 2011). Apesar de tais confirmações, é aconselhado que tomadas radiográficas sejam realizadas após o primeiro trimestre da gestação (Kandan et al, 2011).

A terapêutica medicamentosa, muitas vezes necessárias nos procedimentos endodônticos, como o uso de analgésico, anti-inflamatório e antibióticos, é considerado um fator crítico tanto para período de amamentação quanto ao período da gestação, em vista que a maior parte dos fármacos atravessam a barreira placentária e são transferidos ao bebê pelo leite materno. (Ather et al, 2020; Ebrahim et al, 2014; Luc et al, 2012).

Entre os analgésicos e anti-inflamatórios, é considerado de primeira escolha o uso do paracetamol tanto para o período de amamentação quanto a gestação (Ather et al, 2020). No entanto, o uso de AAS e Ibuprofeno não são recomendados ao período gestacional, principalmente ao terceiro trimestre, devido a capacidade de inibição da síntese de prostagladina, que pode gerar fechamento prematuro dos ductos arteriais do feto, complicações no parto e hemorragia antepartum/pospartum materno/fetal e prolongamento do trabalho de parto (Amadei et al, 2011; Michalowicz et al, 2008; Zanata et al, 2008). Enquanto que no período de gestação não apresentam grandes riscos, pois analgésicos e anti-inflamatórios, geralmente, apresentam concentrações pequenas no leite materno, sendo bem abaixo do valor total da dose terapêutica ingerido pela mãe. Apesar disso, é aconselhável ingerir a medicação de 30 a 60 minutos após a amamentação ou 3 a 4 horas anterior a próxima mamada (Donaldson \& Goodchild, 2012; Amadei et al, 2011; Lopes et al, 2008).

Em relação aos antibióticos, as penicilinas são consideradas a linha de primeira escolha para gestantes, sendo penicilinas, cefalosporinas, ácido clavulânico, eritromicina (base) e clindamicina consideradas seguras (Ather et al, 2020; Donaldson \& Goodchild, 2012; Zanata et al, 2008). Em casos de pacientes alérgicos a penicilinas também deve ser evitado as cefalosporinas devido sua estrutura semelhante as penicilinas, sendo de primeira escolha os macrolídeos, como clindamicina e eritromicina. A tetracíclica é contraindicada tanto no período de amamentação quanto na gestação, pois apresenta alta totoxicidade, causando uma coloração amarelada nas dentições do bebê, além de estar relacionada a hipoplasia dos ossos e cataratas congênitas (Ather et al, 2020).

É necessário que maiores diretrizes de práticas clínicas sejam realizadas para melhor entendimento dos manejos e implicações da prática endodôntica em pacientes gestantes e em períodos de gestação, trazendo maior segurança ao profissional e ao paciente. Essa revisão integrativa se destaca pela abordagem do tema que é tão escasso e controverso na 
literatura, trazendo conjunto de informações para melhor entendimento sobre o assunto e melhor direcionamento ao profissional.

\section{Conclusão}

O cirurgião dentista deve ter conhecimento específico sobre os principais fatores e cuidados do tratamento endodôntico nos períodos de gestação e amamentação. O tipo de anestésico local, tomadas radiográficas e a terapia medicamentosa são fatores críticos para um tratamento sem riscos a mãe e o bebê.

\section{Referências}

Amadei, S., Carmo, E., Pereira, A., Silveira, V., Rocha, R. (2011). Prescrição medicamentosa no tratamento odontológico de grávidas e lactantes. RGO.Revista Gaúcha de Odontologia 59, 31-7.

Ather, A., Zhong, S., Rosenbaum, A. J., Quinonez, R. B., \& Khan, A. A. (2020). Pharmacotherapy during Pregnancy: An Endodontic Perspective. Journal of endodontics, 46(9), 1185-1194. https://doi-org/10.1016/j.joen.2020.05.024.

Botelho, Diana \& Lima, Vivian \& Barros, Myrna \& Almeida, Janaína. (2019). Odontologia E Gestação: A Importância Do Pré-Natal Odontológico. Sanare Revista de Políticas Públicas. 18. https://dx.doi.org/10.36925/sanarev18i2.1376.

Braz, G., Machado, F. C., Oliveira, A. S., Otenio, C. C. M., Alves, R. T., Ribeiro, R. A. (2010) A experiência de um programa de atenção à saúde bucal no atendimento à gestante. HU Revista 36(4), 324-32.

Cabral, M. C. B., Santos, T. S., Moreira, T. P. (2013). Percepção das gestantes do Programa de Saúde da Família em relação à saúde bucal no município de Ribeirópolis, Sergipe, Brasil. Rev Port Saúde Pública. http://hdl.handle.net/10362/105326.

Catão, C. D. S., Gomes, T. A., Rodrigues, R. Q. F., Soares, R. S. C. (2015). Evaluation of the knowledge of pregnant women about the relat ionship between oral diseases and pregnancy complicat ions. Rev Odontol 44(1), 59-65. http://www.scielo.br/pdf/rounesp/v44n1/1807-2577-rounesp-44-01-00059.pdf.

Codata, L. A. B., Nakama, L., Cordoni-Júnior, L., Higasi, M. S. (2011). Atenção odontológica à gestante: papel dos profissionais de saúde. Ciênc Saúde 16(4), 2297-301. https://doi.org/10.1590/S1413-81232011000400029.

Dalto, B. L., da Silva, C. B., Barbosa, G. B., Alberghini, J. F., Linhares, F. (2017). Tratamento endodôntico em gestantes. Rev. odontol. UNESP 46, 0.

Donaldson, M., \& Goodchild, J. H. (2012). Pregnancy, breast-feeding and drugs used in dentistry. Journal of the American Dental Association (1939), 143(8), 858-871. https://doi-org./10.14219/jada.archive.2012.0290.

Ebrahim, Z. F., Cecília, M., De Oliveira, Q., Siqueira, M. P., Franco, J. B., Mo, S. U. (2014). Tratamento odontológico em gestantes. Science in Health 5(1): 32-44.

Giglio, J. A., Lanni, S. M., Laskin, D. M., Giglio, N. W. (2009). Oral health care for the pregnant patient. J Can Dent Assoc 75(1), 43-8.

Jain, K., \& Kaur, H. (2015). Prevalence of oral lesions and measurement of salivary pH in the different trimesters of pregnancy. Singapore medical journal, 56(1), 53-57. https://doi.org/10.11622/smedj.2015010

Kandan, P. M., Menaga, V., \& Kumar, R. R. (2011). Oral health in pregnancy (guidelines to gynaecologists, general physicians \& oral health care providers). JPMA. The Journal of the Pakistan Medical Association, 61(10), 1009-1014.

Lopes, S., Dezan, C. C., Melo, F. J., Alves-souza, R. A., Sturion, L., Barros, K., et al. (2008). Prescrição de medicamentos e anestesia local para gestantes: conduta de cirurgiões-dentistas de Londrina-PR, Brasil. Revista da Faculdade de Odontologia de Porto Alegre 49, $22-7$.

Luc, E., Coulibaly, N., Demoersman, J., Boutigny, H., \& Soueidan, A. (2012). Enquête sur la prise en charge de la femme enceinte au cabinet dentaire [Dental care during pregnancy]. Schweizer Monatsschrift fur Zahnmedizin $=$ Revue mensuelle suisse d'odonto-stomatologie $=$ Rivista mensile svizzera di odontologia e stomatologia, 122(11), 1047-1063.

Martins, L. O., Pinheiro, R. D. P. S., Arantes, D. C., Nascimento, L. S., Santos-Júnior, P. B. (2013). Assistência odontológica à gestante: percepção do cirurgião-dentista. Dental care for pregnant woman: dental surgeon's perceptions. Revista Pan-Amazônica de Saúde, 4(4), 11-18. https://dx.doi.org/10.5123/S2176-62232013000400002.

Mendes, K. D. S., Silveira, R. C. C. P., Galvão, C. M. (2008) Revisão integrativa: método de pesquisa para a incorporação de evidências na saúde e na enfermagem. Texto Contexto Enferm 17(4),758-64. https://doi.org/10.1590/S0104-07072008000400018.

Miana, T. A., Oliveira, A. S., Ribeiro, R. A., Alves, R. T. (2010). Condição bucal de gestantes: implicações na idade gestacional e peso do recém-nascido. HU Rev 36(3), 189-97. https://periodicos.ufjf.br/index.php/hurevista/article/view/1004.

Michalowicz, B. S., DiAngelis, A. J., Novak, M. J., Buchanan, W., Papapanou, P. N., Mitchell, D. A., Curran, A. E., Lupo, V. R., Ferguson, J. E., Bofill, J., Matseoane, S., Deinard, A. S., Jr, \& Rogers, T. B. (2008). Examining the safety of dental treatment in pregnant women. Journal of the American Dental Association (1939), 139(6), 685-695. https://doi-org. /10.14219/jada.archive.2008.0250.

Ministério da Saúde. (2012). Atenção ao pré-natal de baixo risco. Brasil, Brasília (DF): Cadernos de Atenção Básica, n. 32. 
Research, Society and Development, v. 10, n. 14, e105101421876, 2021

(CC BY 4.0) | ISSN 2525-3409 | DOI: http://dx.doi.org/10.33448/rsd-v10i14.21876

Praetzel, J. R., Ferreira, F. V., Lenzi, T. L. (2010). Percepção materna sobre atenção odontológica e fonoaudiológica na gravidez. Rev Gaúch Odontol 58(2), 155-6. file:///D:/RGO-2007-864.pdf.

Reis, F.G. \& Almeida, J. R. S. (2013). Percepção das gestantes sobre o pré-natal odontológico. Ciênc Saúde Colet 4(1), 5 6-62.

Zanata, R. L., Fernandes, K. B., \& Navarro, P. S. (2008). Prenatal dental care: evaluation of professional knowledge of obstetricians and dentists in the cities of Londrina/PR and Bauru/SP, Brazil, 2004. Journal of applied oral science: revista FOB, 16(3), 194-200. https://doi-org. /10.1590/s167877572008000300006 . 